\title{
Minireview
}

\section{Fishing for the signals that pattern the face}

\author{
Thomas F Schilling and Pierre Le Pabic
}

Address: Department of Developmental and Cell Biology, 4462 Natural Sciences II, University of California, Irvine, CA 92697-2300, USA.

Correspondence: Thomas F Schilling. Email: tschilli@uci.edu

\begin{abstract}
Zebrafish are a powerful system for studying the early embryonic events that form the skull and face, as a model for human craniofacial birth defects such as cleft palate. Signaling pathways that pattern the pharyngeal arches (which contain skeletal precursors of the palate, as well as jaws and gills) are discussed in light of a recent paper in BMC Developmental Biology on requirements for Hedgehog signaling in craniofacial development.
\end{abstract}

See research article http://www.biomedcentral.com/1471-213X/9/59

It has been over a century since the discovery that migratory cells of the neural crest give rise to the craniofacial skeleton, unlike the vertebral and limb skeletons, which are derived from embryonic mesoderm [1]. Despite the extraordinarily high frequency of cleft palate and other craniofacial problems in human birth defects, the genetic control of this part of the skeleton remains mysterious. However, recent identification of the genetic basis for many craniofacial syndromes, combined with functional studies in animal models, are beginning to illuminate the cellular and molecular mechanisms underlying skull development and its origins in the neural crest. Studies over the past few years, including one by Schwend and Ahlgren [2] published recently in BMC Developmental Biology, have taken advantage of the availability of zebrafish mutations that disrupt the skull to uncover genes that control its patterning in the early embryo. Moreover, advances in the ability to create transgenic zebrafish have opened up new avenues for following neural crest cells in living embryos and thereby elucidating how genes control skull morphogenesis.

\section{Building the ventral skull: anterior-posterior patterning}

A fundamental feature of the head is its modular organization - the embryonic hindbrain and the pharynx become partitioned into a series of segments. Within 24 hours postfertilization the zebrafish hindbrain has become segmented into seven rhombomeres and the tissues surrounding the pharynx subdivide into seven so-called arches (Figure 1a). These include the mandibular arch (arch 1), which forms the jaw, the hyoid $\operatorname{arch}(\operatorname{arch} 2)$, which forms the jaw support, and five gill arches (arches 3-7). All have a similar structure, including skeletal, neural and glial components derived from neural crest, muscles and blood vessels derived from mesoderm, and an outer sheath of surface ectoderm and endoderm.

These similarities led early comparative anatomists to argue that jaws, which form from the prominent mandibular arch, evolved from an ancestral gill arch (reviewed in [3]). Consistent with this idea, early vertebrates had pharyngeal arches but were jawless - arches are one of the most ancient vertebrate traits, found even in nonvertebrate chordates such as amphioxus. Evidence to support the gill-arch origin for jaws has come with the discovery of similar patterns of gene expression in every arch in the series. For example, recent studies in a chondrichthyan fish, a skate, suggest that the network of secreted growth factors found in the mandibular arch, which includes Sonic Hedgehog (Shh; see below), fibroblast growth factor and retinoic acid, is conserved in the gill arches, and inhibitors of Shh signaling disrupt skeletal patterning similarly in every arch [4].

Surprisingly, however, recent genetic evidence argues against the model of a gill-arch ground plan and instead suggests that the default state for an arch is more mandibular in character. Combinatorial expression of Hox genes underlies arch-specific morphologies, with the exception of the mandibular arch, which is devoid of Hox activity. This 'Hox code' applies to all non-mandibular arches (there are four arches in mammals but up to nine in other vertebrates) and in each case posterior arches acquire more anterior characteristics in the absence of Hox gene function. For instance genetic knockout of Hoxa2 in mice, or combined loss of hoxa2 and hoxb2 in zebrafish, leads to a replacement of second-arch-derived structures by those of the first arch [5,6]. Strikingly, Minoux et al. [7] recently showed that all posterior arches take on a mandibular identity following the conditional loss of all Hoxa cluster genes in mouse cranial neural crest cells. Thus, posterior arch identity must override a default 'mandibular' program that dominates in the absence of Hox expression. 


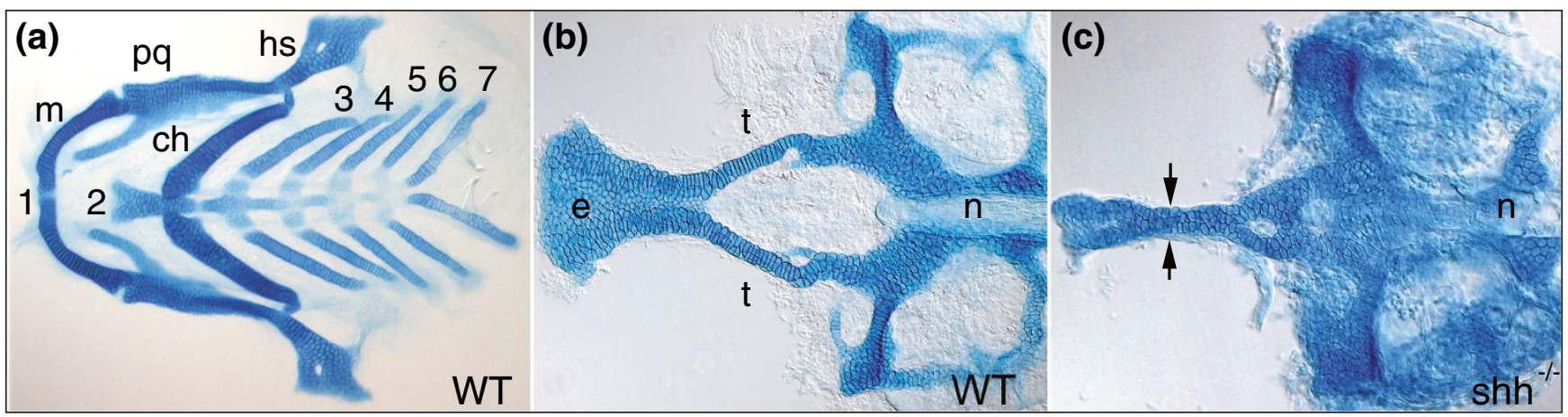

Figure 1

Pharyngeal arch and neurocranial cartilage patterns in wild-type and Hh-deficient zebrafish larvae. Alcian-stained cartilages were dissected and flat mounted; dorsal views are shown, anterior to the left. (a) The wild-type (WT) pharyngeal arches at 4.5 days postfertilization include: the mandibular arch (arch 1), containing Meckel's cartilage $(\mathrm{m})$ and the palatoquadrate (pq); the hyoid arch (arch 2), containing the ceratohyal (ch) and hyosymplectic (hs); and five more posterior, branchial arches (3-7), each containing a ceratobranchial cartilage. (b) The wild-type neurocranium at 4.5 days postfertilization includes paired trabeculae (t) and an ethmoid plate (e). (c) Neurocranial defects in sonic hedgehog (shh) mutants. Trabecular cartilages fuse (arrows) at the midline. e, ethmoid; $n$, notochord; t, trabecula. Panels (b) and (c) taken from [19].

\section{Epithelial-mesenchymal interactions pattern the arches}

Once head segmentation is established, the next critical step is differential growth and patterning within each arch. In most vertebrates the mandibular arch is much larger than the others. Gill arches are prominent structures in aquatic vertebrates such as fish, but are only transiently present in mammals and later become incorporated into the throat and larynx. How does the identity acquired through the Hox code result in such large differences in arch morphogenesis?

The answer appears to lie in how neural crest cells within the arch respond to signals from surrounding epithelia. Early grafting studies in avian embryos demonstrated that mandibular neural crest transplanted posteriorly gave rise to an ectopic beak and other mandibular structures [8], suggesting that similar signals exist in both anterior and posterior arches, and that segmental identity is intrinsic to neural crest cells. More recent variations on these types of grafts suggest that this is only part of the story $[9,10]$. Nevertheless, further evidence for an intrinsic Hox code in the neural crest has come from studies of zebrafish $\mathrm{moz}$ mutants, which disrupt an essential activator of Hox paralog group 2 gene expression and exhibit transformations of the hyoid (arch 2) to a mandibular ( $\operatorname{arch} 1$ ) identity, similar to Hoxa2/b2 double morpholino-injected (morphants) $[6,11]$. Mosaic studies combining mutant and wild-type cells suggest that Moz and Hox paralog group 2 proteins act cell-autonomously in the second arch skeletogenic neural crest cells - without them skeletal elements acquire the size and shape of their counterparts in the mandibular arch [12]. Thus, Hox genes appear to instruct neural crest cells to interpret arch signals appropriately.
These signals largely come from the endodermal and/or ectodermal epithelia that ensheath each arch. Here again, mutational studies and transgenics in zebrafish have been very informative. Time-lapse movies of fluorescent transgenes that mark skeletogenic neural crest cells reveal that early cartilage formation occurs in close proximity to the endodermal 'pouches' that separate adjacent arches - these are highly conserved from fish to humans. Zebrafish mutants that lack endoderm fail to form cartilage, and restoring endoderm rescues skeletogenesis [13]. Mutants that disrupt individual pouches disrupt adjacent cartilages, presumably because of the loss of chondrogenic signals emitted by the pharyngeal endoderm $[14,15]$. One secreted signaling molecule expressed by this endoderm and required for craniofacial development is Shh, a vertebrate relative of the product of the Drosophila segment polarity gene hedgehog.

\section{Requirements for Hedgehog signaling in the face}

Hh signaling influences multiple aspects of craniofacial development. In humans, disruption of SHH, as well as several other components of the Hh signal transduction pathway, causes holoprosencephaly (HPE), which is among the most common craniofacial birth defects, characterized by cyclopia, midfacial clefting and arch defects [16]. Conversely, ectopic expression of Shh in chick embryos causes complete duplications of the mandible [17]. Hh signaling impinges on multiple cell types, including pharyngeal endoderm, surface ectoderm, ventral brain and neural crest. Much of what is known centers around the midline - fusion or loss of midfacial bones are typical of HPE patients, as well as of Shh-deficient mice or zebrafish (Figure 1b,c). New insights came with the knockout of the 
essential Hh co-receptor, Smoothened (Smo), which when removed specifically from neural crest cells causes similar craniofacial defects in mice as the knockout [18]. Many outstanding questions remain, however, including the location of the sources of Shh and its relatives in the face, the precise timing of their actions, and how defects in $\mathrm{Hh}$ signaling cause such variable defects (for example, midline fusions versus cleft palate).

Here again, zebrafish offer the advantage of a forward genetic approach and a large collection of mutants in the Hh signaling pathway. Fate-mapping studies in both Shh and Smo mutants, using transgenics that label migrating cranial neural crest cells, have revealed that Hh signaling plays two distinct roles in patterning the cartilages of the primary palate: one in neural crest cells in the maxillary region of the first arch, and the second in a more anterior population of neural crest cells that forms the ethmoid plate in the midline [19]. Disruption of Hh signaling leads to defects in one or both populations, resulting in cyclopia (when maxillary regions fuse; Figure 1c) or mid-facial clefting (when the midline ethmoid is not induced), and treatment with the alkaloid cyclopamine, which chemically blocks Smo function, only disrupt one or the other of these populations, depending on the stage of treatment. Shh expressed in the ventral forebrain and oral ectoderm appears to be the critical source in the midline $[19,20]$. These results exemplify the complex spatial and temporal nature of Hh signaling in the face, and help explain why human HPE manifests itself in so many different ways.

Hh signaling also appears to have distinct roles in the mandibular and more posterior arches. Schwend and Ahlgren [2] show that chameleon (con) mutants, which lack a fully functional Dispatched (Disp1) protein crucial for Hh secretion, develop maxillary fusions, loss of jaw joints and a complete lack of cartilage in arches 3-7. disp1 co-localizes with Shh in pharyngeal epithelia shortly after neural crest migration into the arches. Defects in the mandibular arch are not surprising, though the results in this paper hint at a previously unappreciated role for $\mathrm{Hh}$ signaling in jaw joint development. What is more surprising is the selective loss of expression of some genes involved in skeletogenesis (soxga, $d l \times 2 a$ ) and not others (for example, soxgb, hand2) in the gill arches in con/disp1 mutants. In zebrafish, defects in the gill arches are often secondary consequences of developmental delay or heart defects. However, this is clearly not the case in con/disp1 mutants and instead, fibrous connective tissue appears to replace cartilage. Thus, Hh signaling is required for cartilage differentiation in the posterior arches, and several lines of evidence suggest that this is distinct from its roles in the palate or mandible. Such specific regulation of gene expression and skeletogenesis by Hh may offer insights into the nature of defects in other zebrafish mutants that affect the posterior arches [21].

\section{Deciphering the secrets of the throat}

Given that Shh and other signals are expressed in similar patterns in different arches, one big open question is how the responses to these signals are modulated in a segmentspecific manner. Why is the mandible in a fish so much larger than its gills? We still know relatively little about the targets of Hox genes within different segments, or how the combinatorial Hox code is interpreted to give different morphologies. What seems clear is that not all cells within a segment necessarily interpret the code in the same fashion, nor do the signals impinging upon them have a single effect on cell behavior. For example, Shh appears to have at least two roles in the face, one in establishing the patterns of palate or joint precursors and one in cartilage differentiation, both in the palate and in the gills. Whether this is truly a dual role for Shh or reflects distinct roles for its relatives, such as Indian hedgehog (Ihh), remains unclear.

Zebrafish provide the opportunity to address these questions more directly than is possible in a chick or mouse, as we can watch neural crest and arch development in real time using transgenics, taking advantage of the transparency of the live fish embryos. The added ability to screen for craniofacial mutants in zebrafish is proving fruitful for discovering novel factors involved in arch development. These are candidates for genes mutated in as yet unidentified human craniofacial syndromes. The importance of this approach is apparent when considering that craniofacial defects are so common (for example, 1 in 700 live births have cleft lip or palate), yet the genetic basis for most is unknown. Most of the genes known to be involved have roles in embryonic development, arguing that as new genetic causes are revealed, their functions can be rapidly evaluated in the context of the known pathways that pattern the arches. Craniofacial research is now undergoing a rapid expansion, with the accelerated identification of human disease genes and new model systems for functional analysis, and the zebrafish promises to be a central player.

\section{Acknowledgements}

Craniofacial research in the Schilling lab is funded by the NIH - R01 DE13828.

\section{References}

1. Platt J: Ectodermic origin of the cartilages of the head. Anat Anz 1893, 8:506-509.

2. Schwend T, Ahlgren SC: Zebrafish con/disp1 reveals multiple spatiotemporal requirements for Hedgehog-signaling in craniofacial development. BMC Dev Biol 2009, 9:59.

3. Northcutt RG: Historical hypotheses regarding segmentation of the vertebrate head. Integr Comp Biol 2008, 48:611619.

4. Gillis JA, Dahn RD, Shubin NH: Shared developmental mechanisms pattern the vertebrate gill arch and paired fin skeletons. Proc Natl Acad Sci USA 2009, 106:5720-5724.

5. Rijli FM, Mark M, Lakkaraju S, Dierich A, Dolle P, Chambon P: A homeotic transformation is generated in the rostral 
branchial region of the head by disruption of HoxA-2, which acts as a selector gene. Cell 1993, 75:1333-1349.

6. Hunter MP, Prince VE: Zebrafish Hox paralogue group 2 genes function redundantly as selector genes to pattern the second pharyngeal arch. Dev Biol 2002, 247:367-389.

7. Minoux M, Antonarakis GS, Kmita M, Duboule D, Rijli FM: Rostral and caudal pharyngeal arches share a common neural crest ground pattern. Development 2009, 136:637645.

8. Noden DM: The embryonic origins of avian cephalic and cervical muscles and associated connective tissues. Am J Anat 1983, 168:257-276.

9. Trainor P, Krumlauf R: Plasticity in mouse neural crest cells reveals a new patterning role for cranial mesoderm. Nat Cell Biol 2000, 2:96-102.

10. Couly G, Creuzet S, Bennaceur S, Vincent C, Le Douarin NM: Interactions between Hox-negative cephalic neural crest cells and the foregut endoderm in patterning the facial skeleton in the vertebrate head. Development 2002, 129: 1061-1073.

11. Miller $C T$, Maves $L$, Kimmel CB: moz regulates Hox expression and pharyngeal segmental identity in zebrafish. Development 2004, 131:2443-2461.

12. Crump JG, Swartz ME, Eberhart JK, Kimmel CB: Mozdependent Hox expression controls segment-specific fate maps of skeletal precursors in the face. Development 2006, 133:2661-2669.

13. David NB, Saint-Etienne L, Tsang M, Schilling TF, Rosa FM: Requirement for endoderm and FGF3 in ventral head skeleton formation. Development 2002, 129:4457-4468.

14. Crump JG, Maves L, Lawson ND, Weinstein BM, Kimmel CB: An essential role for Fgfs in endodermal pouch formation influences later craniofacial skeletal patterning. Development 2004, 131:5703-5716.
15. Crump JG, Swartz ME, Kimmel CB: An integrin-dependent role of pouch endoderm in hyoid cartilage development. PLoS Biol 2004, 2:1432-1445.

16. Roessler E, Belloni E, Gaudenz K, Jay P, Berta P, Scherer SW, Tsui LC, Muenke M: Mutations in the human Sonic Hedgehog gene cause holoprosencephaly. Nat Genet 1996, 14: 357-360.

17. Brito JM, Teillet MA, Le Douarin NM: Induction of mirrorimage supernumerary jaws in chicken mandibular mesenchyme by Sonic Hedgehog-producing cells. Development 2008, 135:2311-2319.

18. Jeong J, Mao J, Tenzen T, Kottman AM. McMahon AP: Hedgehog signaling in the neural crest cells regulates the patterning and growth of facial primordia. Genes Dev 2004, 18:937-951.

19. Wada N, Javidan Y, Nelson S, Carney TJ, Kelsh RN, Schilling TF: Hedgehog signaling is required for cranial neural crest morphogenesis and chondrogenesis at the midline in the zebrafish skull. Development 2005, 132:3977-3988.

20. Eberhart JK, Swartz ME, Crump JG, Kimmel CB: Early Hedgehog signaling from neural to oral epithelium organizes anterior craniofacial development. Development 2005 , 133:1069-1077.

21. Schilling TF, Piotrowski T, Grandel $H$, Brand $M$, Heisenberg $C P$, Jiang YJ, Beuchle D, Hammerschmidt M, Kane DA, Mullins MC, van Eeden FJ, Kelsh RN, Furutani-Seiki M, Granato M Haffter P, Odenthal J, Warga RM, Trowe T, Nüsslein-Volhard C: Jaw and branchial arch mutants in zebrafish I: branchial arches. Development 1996, 123:329-344.

Published: 22 December 2009

doi:10.1186/jbiol205

(C) 2009 BioMed Central Ltd 